\title{
A new design of high output voltage rectifier for rectenna system at $2.45 \mathrm{GHz}$
}

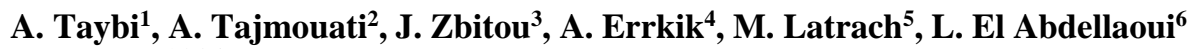 \\ 1,2,3,4,6LMEET, FST of Settat, Hassan 1st University, Settat, Morocco \\ ${ }^{5}$ Microwave Group ESEO Angers, France
}

\begin{tabular}{l}
\hline Article Info \\
\hline Article history: \\
Received Aug 29, 2018 \\
Revised Nov 15, 2018 \\
Accepted Nov 26, 2018 \\
\hline
\end{tabular}

Keywords:

Microstrip rectifier

Output voltage

Schottky diode

Stepped-impedance low pass

filter

Wireless power transmission

\begin{abstract}
This paper deals with the design and achievement of a novel microstrip rectifier with high conversion efficiency and output voltage. Firstly, we have designed a rectifier based on HSMS2820 Schottky diodes by using a series topology to convert the electromagnetic energy into DC power. Then, a stepped-impedance low pass filter was implemented to filter the unwanted harmonics generated by the non-linear Schottky diode. Both of the structures have been simulated and fabricated on an FR4 substrate with dielectric permittivity constant 4.4 , thickness of $1.6 \mathrm{~mm}$ and loss tangent of 0.025 . Good performances were confirmed throughout the measurement results and an interesting output voltage was observed.
\end{abstract}

Copyright $@ 2019$ Institute of Advanced Engineering and Science. All rights reserved.

\section{Corresponding Author:}

A. Taybi,

LMEET, FST of Settat,

Hassan 1st University, Settat, Morocco.

Email: taybi.abdellah@gmail.com

\section{INTRODUCTION}

Owing to the progress of electronics in recent years, it has become increasingly common to use sensors, sensor networks and wireless actuators in many areas (space, military, medical, domestic, ...), and especially in places that are dangerous and difficult to access. For a better integration in the environment, it is necessary to reduce the size of these systems and to ensure their energy autonomy. Conventional battery or battery powering techniques remain restrictive or even difficult to match for certain applications. Indeed, they are limited in autonomy, require periodic replacements and their recycling is expensive. Recently, as a solution, various wireless power technologies are proposed in [1-4] which have generated significant research interest in this area. The concept is to eliminate in the future the constraints of battery replacement.

The aim of this paper is to propose an alternative solution that is efficient, less restrictive and more respectful of the environment. From this point of view, wireless power transmission (WPT) [5] is a very interesting solution. As shown in Figure 1(a), it consists of transmitting energy from one point to another through the free space, and once captured and converted into DC, will be used to feed the wireless device(s). In this study, we focused on microwave energy transfer at $2.45 \mathrm{GHz}$ which is the Industrial Scientific Medical (ISM) frequency band. Firstly, the DC electrical energy is converted into microwave energy using an RF source. Then, this energy is radiated in the free space by a transmitting antenna. Finally, the radiated energy is captured by a Rectenna (Rectifying Antenna) [6-7] circuit, converted into DC power and delivered to a resistive load.

A conventional rectenna circuit (Figure 1(b)) is composed from a receiving antenna [8-11] followed by a non-linear characteristic RF-DC conversion circuit. This circuit contains most often one or more Schottky diodes [12], an HF input filter, a DC output filter and a resistive load. The challenge is to optimize 
the entire rectenna, with the objective of maximizing the DC output in terms of RF-DC conversion efficiency. The optimization must be done on the whole circuit, hence the need to use global analysis methods combining electromagnetic simulation and circuit. Numerical modeling will be a fundamental tool throughout this work, the aim being to take into account all the possible couplings between the different parts of the circuit.

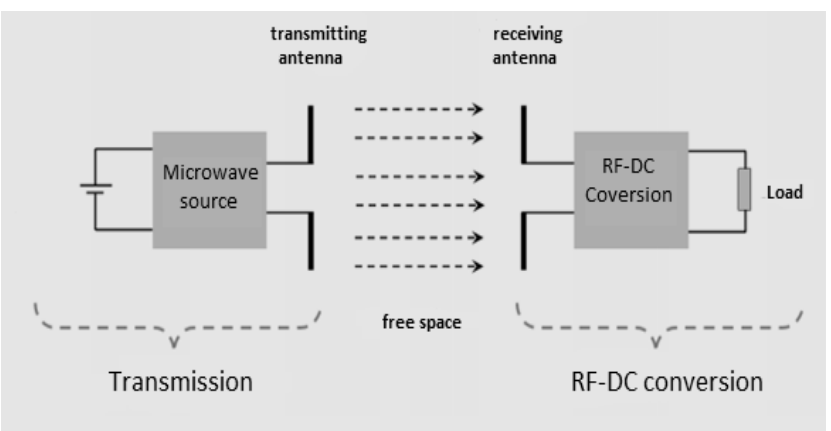

(a)

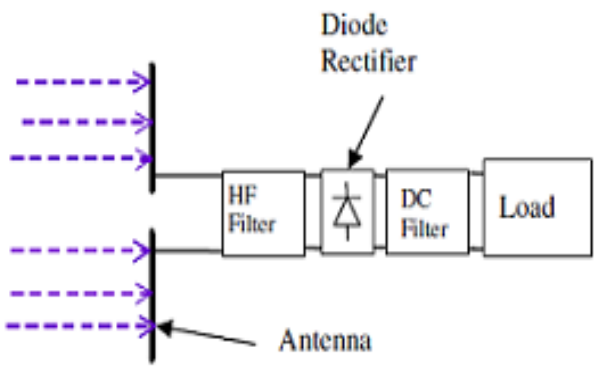

(b)

Figure 1. (a) Wireless power transmission system, (b) Block diagram of rectenna circuit [13]

The aim from this work is to design, optimize, realize and experimentally characterize innovative, compact and high voltage rectifier circuit. A series topology is used and a stepped-impedance Low Pass Filter LPF is placed at the output of the circuit to obtain a stable DC output voltage.

\section{RECTIFIER DESIGN AND SIMULATION}

The simulation and optimization of the circuit were carried out under Advanced Design System ADS [14] solver with a coupling between harmonic balance and Large-signal S-parameters. Prior to the optimization step, parametric studies were conducted to determine the sensitivity of the conversion efficiency to some important parameters and more particularly, the load $\left(\mathrm{R}_{\mathrm{L}}\right)$, the diode parameters, the capacity of the DC filter, the microstrip line sections. The results that emerge from this study allowed us to make certain choices and to simplify the optimization process.

\subsection{Mono-Diode Series Rectifier}

The developed circuit is in series topology (Figure 2(a)), it contains a Schottky diode HSMS 2820 which has a low junction capacity $\mathrm{Cj} 0(0.18 \mathrm{pF})$, a low RS series resistance $(5 \Omega)$, a junction resistance RJ, a package inductance LP, and a package capacitance CP. Its forward-bias turn-on voltage VBI and breakdown voltage VB. The equivalent electrical model of the diode is given in Figure 2(b). The circuit is powered by a characteristic impedance $50 \Omega$ microstrip line, it has been etched on the R4 substrate $(\varepsilon r=4.4$, $\mathrm{h}=1.6 \mathrm{~mm}, \tan \delta=0.025)$.

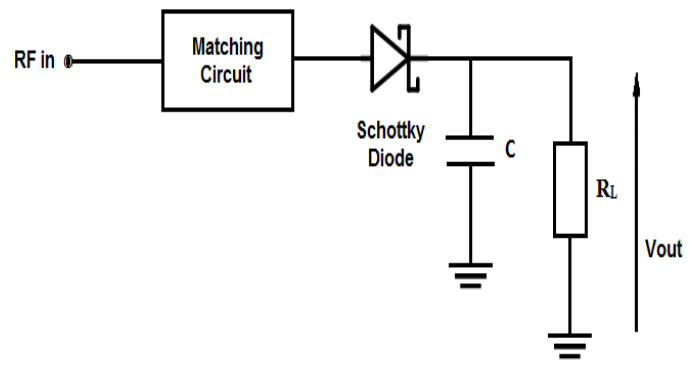

(a)

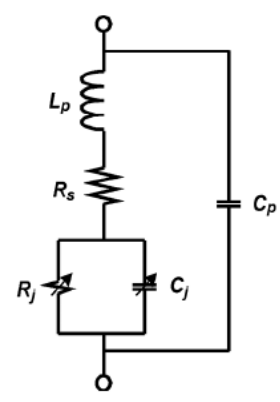

(b)

Figure 2. (a) The proposed rectifier topology (b) Equivalent circuit model of the Schottky diode 
The efficiency [15] describes the ability of the rectifier to provide continuous electrical power to the load from the RF energy provided by the receiving system or other microwave power source. This efficiency represents the main objective during a process of the conversion circuit optimization. The variation of the Schottky diode impedance generates a mismatch that directly affects the conversion efficiency. Figure 3 shows the simulated results of the proposed rectifier, the circuit has a good matching input impedance around $2.45 \mathrm{GHz}$ in the ISM band. The sensitivity of the rectifier is directly related to the sensitivity of the diodes used and its non-linear characteristic. The following sections will describe the methodology and the different steps followed to optimise the proposed rectifier associated to the DC filter.

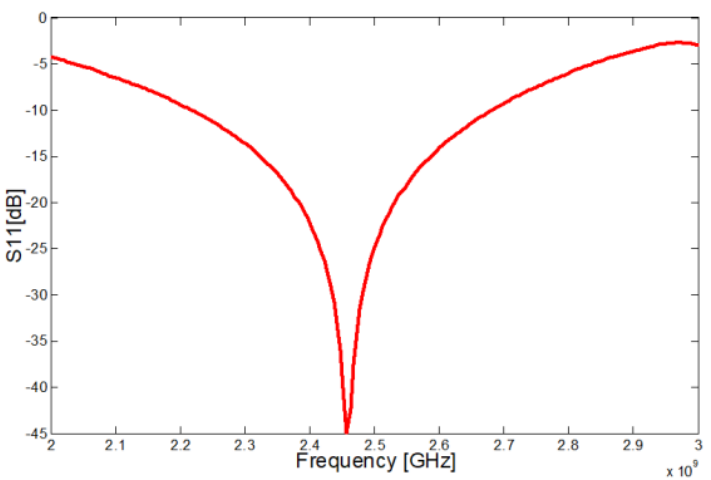

(a)

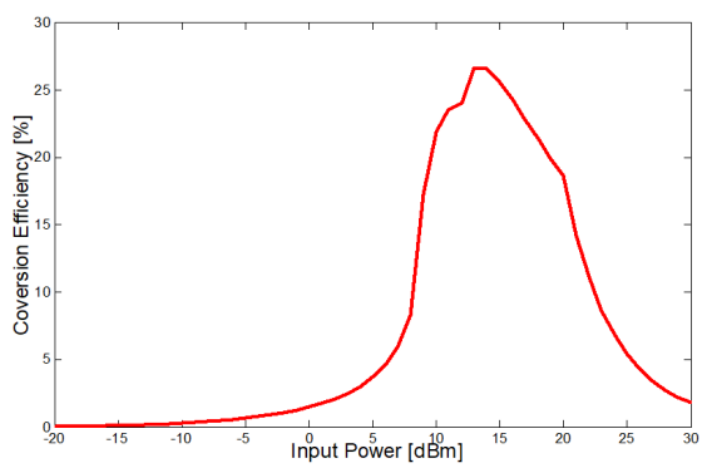

(b)

Figure 3. Simulated results (a) S11 versus frequency, (b) Conversion efficiency versus input power

\subsection{Stepped-Impedance Low-Pass Filter}

Passive microwave filters are very important part of modern telecommunication systems (Rectenna applications). The technology of microwave filters made the evidence, from the point of view of design and miniaturization, as well as from the point of view of the use of specific materials, in order to achieve better responses of selectivity, quality factor and tunability in frequency. Improved electrical performances, increased selectivity, a compact size and reduced production costs are the main constraints facing the design of RF and microwave filters. In this context, the development of highly selective filters, with low levels of losses and compact size, is at present a field of activity of fundamental interest. Such filters are made by various technologies, microstrip lines, slot lines and coplanar waveguides. Microstrip [16] is one of the dominant technologies, which can provide implementation in low profile topologies.

An efficient way to implement low pass filters in microstrip technology is to use alternating sections of very high and very low characteristic impedance lines. Such filters are usually referred as stepped impedance filters. This kind of filters are popular because they are easier to design and take up less space than a similar low-pass filter using stubs. The stepped impedance filter is one of the conventional filters, mainly due to the ease of implementation in either microstrip or coplanar technology. This filter is normally composed of alternating low and high impedance regions (the high impedance lines act as series inductors and the low impedance lines act as shunt capacitors), where the change in impedance is controlled by the width of the strip. For achieving a high degree of attenuation in the stop band it is necessary to obtain a high to low impedance ratio $\left(\mathrm{Z}_{\mathrm{H}} / \mathrm{Z}_{\mathrm{L}}\right)$ or to increase the order of the filter. Also, one of the main requirements for the stepped impedance filter is that each section must be less than half of the quarter wavelength at the cutoff frequency $(1 \lambda / 8)$. The overall size of the filter will be large at low frequencies especially if the number of the elements is increased to achieve special requirements as in sharp edge filters.

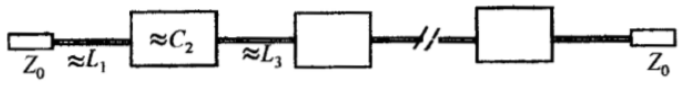

(a)

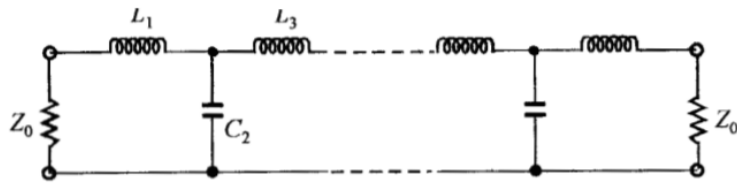

(b)

Figure 4. (a) General structure of the stepped-impedance low pass microstrip filters. (b) L-C ladder type of low pass filters to be approximated. 
Figure 4(a) shows a general structure of the stepped-impedance low pass microstrip filters, which use a cascaded structure of alternating high and low impedance transmission lines. These are much shorter than the associated guided wavelength, so as to act as semi lumped elements. The high-impedance lines act as series inductors and the low-impedance lines act as shunt capacitors. Therefore, this filter structure is directly realizing the L-C ladder type of low pass filters of Figure 4(b). Some a priori design information must be provided about the microstrip lines, because expressions for inductance and capacitance depend upon both characteristic impedance and length. It would be practical to initially fix the characteristic impedances of high- and low-impedance lines by consideration of:

a) $\mathrm{Z}_{\mathrm{L}}<\mathrm{Z}_{0}<\mathrm{Z}_{\mathrm{H}}$, where $\mathrm{Z}_{\mathrm{L}}$ and $\mathrm{Z}_{\mathrm{H}}$ denote the characteristic impedances of the low and high impedance lines, respectively, and $Z_{0}$ is the source impedance, which is usually $50 \mathrm{ohms}$ for microstrip filters.

b) A lower $Z_{\mathrm{L}}$ results in a better approximation of a lumped-element capacitor, but the resulting line width $\mathrm{W}_{\mathrm{C}}$ must not allow any transverse resonance to occur at operation frequencies.

c) A higher $Z_{\mathrm{H}}$ leads to a better approximation of a lumped-element inductor, but $\mathrm{Z}_{\mathrm{H}}$ must not be so high that its fabrication becomes inordinately difficult as a narrow line, or its current-carrying capability becomes a limitation.

In this section, a stepped-impedance Low-Pass Filter is designed and simulated. It's about a LPF having a maximally flat response and a cut-off frequency of $1.5 \mathrm{GHz}$. It is desired to have more than $20 \mathrm{~dB}$ insertion loss at $2.5 \mathrm{GHz}$. The filter impedance is $50 \Omega$ the highest practical line impedance is $120 \Omega$, and the lowest is $20 \Omega$. Consider the effect of losses when this filter is implemented with an FR4 substrate and copper conductors. To design the desired filter, we must first define the appropriate order by using the following Figure 5.

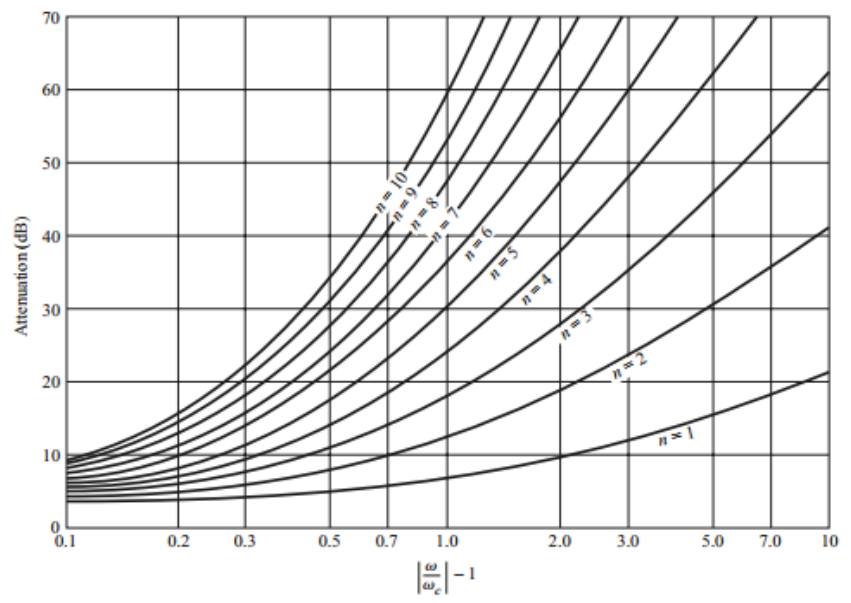

Figure 5. Attenuation versus normalized frequency for maximally flat filter prototypes [17]

Then, the element values for the ladder-type circuits of Figure 4(b) can be tabulated. Table 1 gives such element values for maximally flat low-pass filter prototypes for $\mathrm{N}=1$ to 10 . These data can be used with either of the ladder circuits of Figure 4(b) in the following way.

Table 1: Element Values for Maximally Flat Low-Pass Filter Prototypes $(g 0=1, \omega c=1, N=1$ to 10 ).

\begin{tabular}{|c|c|c|c|c|c|c|c|c|c|c|c|}
\hline $\mathbf{N}$ & $g_{1}$ & $g_{2}$ & $\mathbf{g}_{3}$ & $\mathrm{~g}_{4}$ & g5 & g6 & g7 $_{7}$ & g8 & g9 & $\mathrm{g}_{10}$ & $g_{11}$ \\
\hline 1 & 2.0000 & 1.0000 & & & & & & & & & \\
\hline 2 & 1.4142 & 1.4142 & 1.0000 & & & & & & & & \\
\hline 3 & 1.0000 & 2.0000 & 1.0000 & 1.0000 & & & & & & & \\
\hline 4 & 0.7654 & 1.8478 & 1.8478 & 0.7654 & 1.0000 & & & & & & \\
\hline 5 & 0.6180 & 1.6180 & 2.0000 & 1.6180 & 0.6180 & 1.0000 & & & & & \\
\hline 6 & 0.5176 & 1.4142 & 1.9318 & 1.9318 & 1.4142 & 0.5176 & 1.0000 & & & & \\
\hline 7 & 0.4450 & 1.2470 & 1.8019 & 2.0000 & 1.8019 & 1.2470 & 0.4450 & 1.0000 & & & \\
\hline 8 & 0.3902 & 1.1111 & 1.6629 & 1.9615 & 1.9615 & 1.6629 & 1.1111 & 0.3902 & 1.0000 & & \\
\hline 9 & 0.3473 & 1.0000 & 1.5321 & 1.8794 & 2.0000 & 1.8794 & 1.5321 & 1.0000 & 0.3473 & 1.0000 & \\
\hline 10 & 0.3129 & 0.9080 & 1.4142 & 1.7820 & 1.9754 & 1.9754 & 1.7820 & 1.4142 & 0.9080 & 0.3129 & 1.0000 \\
\hline
\end{tabular}


The element values are numbered from $g_{0}$ at the generator impedance to $g_{N+1}$ at the load impedance for a filter having $\mathrm{N}$ reactive elements. To replace the series inductors and shunt capacitors with sections of low-impedance and high-impedance lines, the required electrical line lengths, $\beta i$, along with the physical microstrip line widths, $\mathrm{Wi}$, and lengths, $\mathrm{Li}$, are given by :

$\beta I=\frac{L R_{0}}{Z_{H}} \quad$ (Inductor)

$\beta I=\frac{C Z_{L}}{R_{0}} \quad$ (Capacitor)

The final design of the stepped-impedance low-pass filter is shown in the following Figure 6.

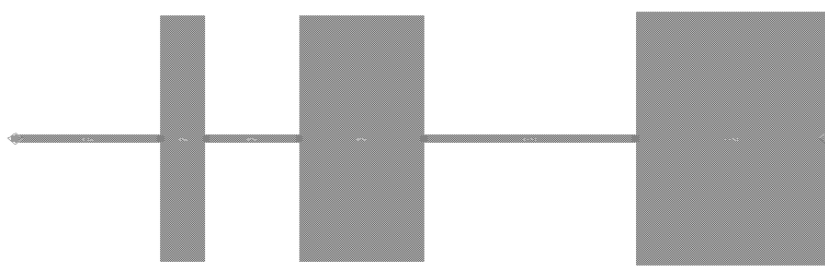

Figure 6. The proposed filter layout

Figure 7 shows a good response of the filter in rejecting the undesired frequency bands (higher order harmonics generated by the schottky diode).
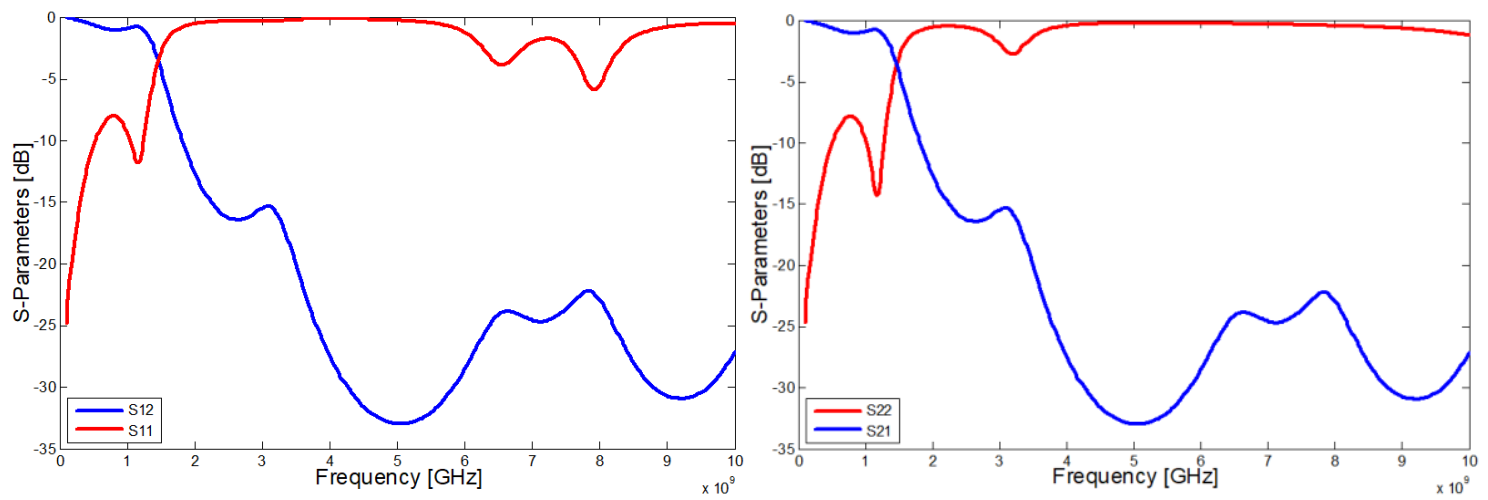

Figure 7. Simulated filter response versus frequency

\subsection{Final Rectifier Design}

Figure 8 shows the designed rectifier where the initial rectifier structure and the stepped-impedance low pass filter have been associated. A matching circuit is essential in providing the maximum power transfer from the antenna to the rectifier circuit. As it is shown in Figure 8, to match the input impedance of the diode with the antenna impedance of $50 \Omega$ (or any other source of RF power), a combination of a transmission line and a microstrip radial stub is designed. The optimization of the impedance matching consists of adjusting the length and the angle of the microstrip radial stub and the length of the transmission line by using an optimization method under ADS software. In addition, the same Schottky diode mentioned in the initial rectifier design section have been employed in the DC portion of the rectifier, in series with $\mathrm{R}_{\mathrm{L}}$ [18] in order to improve the detection sensitivity, so that the diode acts as a variable resistor: 


$$
R_{j}=n K T / q\left(I_{s}-I_{b}\right)
$$

Where $n$ is the diode ideality factor, $\mathrm{K}$ is the Boltzmann's constant, $\mathrm{q}$ is the electronic charge, $\mathrm{I}_{\mathrm{s}}$ is the diode saturation current, $\mathrm{I}_{\mathrm{b}}$ is the external bias current, and $\mathrm{T}$ is the temperature of the diode in degrees Kelvin.

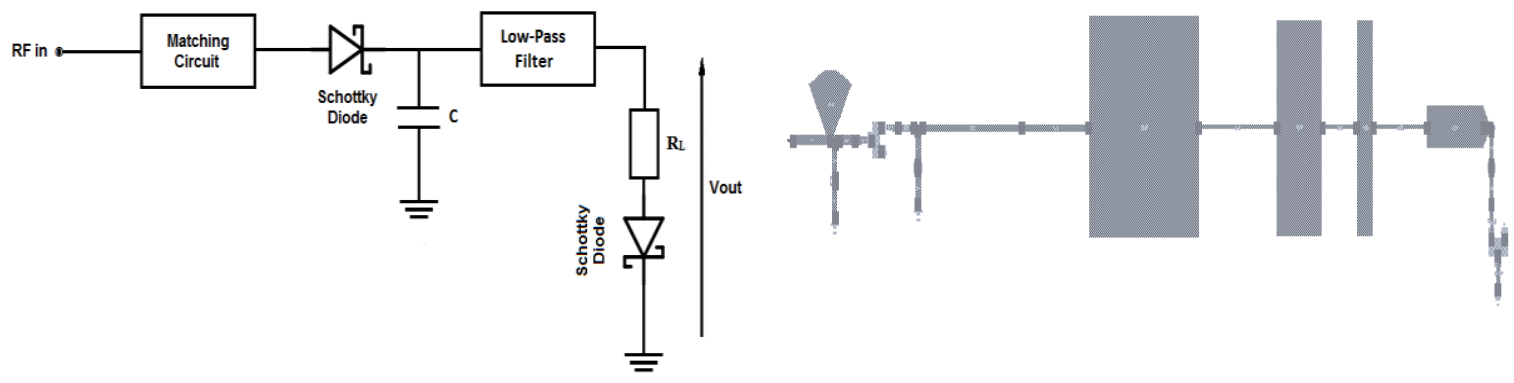

Figure 8. Final rectifier design

The combination of passive elements (antennas, line sections, inductors, capacitors) and active elements (diodes) poses the problem of the overall numerical simulation of the device. Regarding the use of high frequency circuits, it is important to select a suitable simulation environment, which is able to offer different types of simulations specific to RF circuits. Advanced Design Systems from Agilent Technologies offers a broad spectrum of tools dedicated to the design of RF circuits. Time (Transient), frequency (Harmonic Balance, SParameter, LSSP) and electromagnetic (Momentum, EMDS) simulations are included in the software. In addition, a large library of component models such as Schottky diodes is available. For the designed rectifier, simulations have been performed by using Harmonic Balance as a time-frequency analysis of the non-linear behaviour of the circuit. Moreover, the matching input impedance is validated by using Large Signal S-Parameters (LSSP).

The efficiency $(\eta)$ of the microwave rectifier is defined by:

$$
\eta=P_{d c} / P_{r}=V_{d c}^{2} / P_{r} R_{L}
$$

Where $P_{D C}$ is the dc power produced at the load resistance $\left(R_{L}\right)$ of the rectifier and $P_{r}$ is the power received at the antenna of rectenna or any other source of microwave energy. $\mathrm{P}_{\mathrm{r}}$ is calculated from the Friis transmission equation wich gives the amount of power an antenna received under ideal conditions from another antenna. The Power from isotropic antenna falls off as $\mathrm{R}^{2}$, so that the power density (p) would be:

$$
\mathrm{p}=\mathrm{p}_{\mathrm{t}} / 4 \pi \mathrm{R}^{2}
$$

Multiplying by the gain of the transmitting antenna gives a real antenna pattern

$$
\mathrm{p}=\left(\mathrm{p}_{\mathrm{t}} / 4 \pi \mathrm{R}^{2}\right) \mathrm{G}_{\mathrm{t}}
$$

If receiving antenna has an effective aperture of $\mathrm{A}_{\text {eff }}$ the power received by this antenna $\left(\mathrm{P}_{\mathrm{r}}\right)$ is

$$
P_{r}=p \cdot A_{e f f}
$$

Thus

$$
P_{r}=\left(p_{t} / 4 \pi R^{2}\right) G_{t} A_{e f f}
$$

The effective aperture of an antenna can be written as:

$$
A_{e f f}=\left(\lambda^{2} / 4 \pi\right) \cdot G
$$

So, we conclude that the friis transmission equation could be expressed as follow: 
Figure 9 shows the simulation results after optimization of the circuit, it's clear from the graphs that the performances of the rectifier have been remarkably improved.

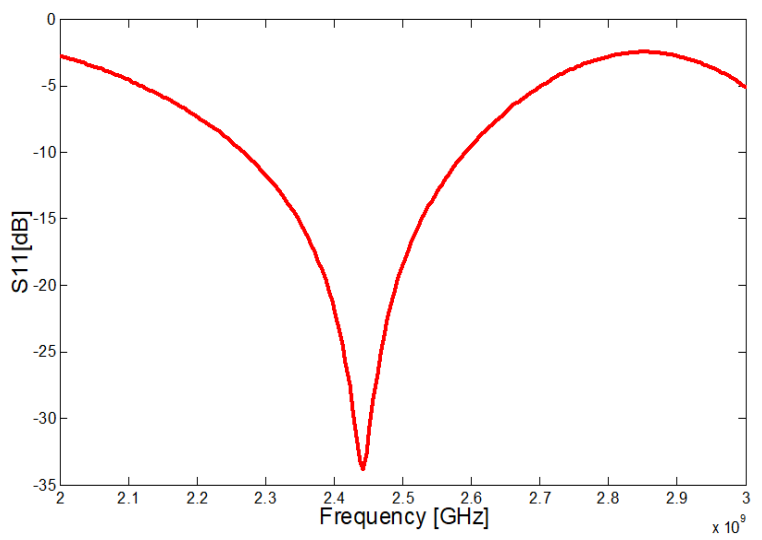

(a)

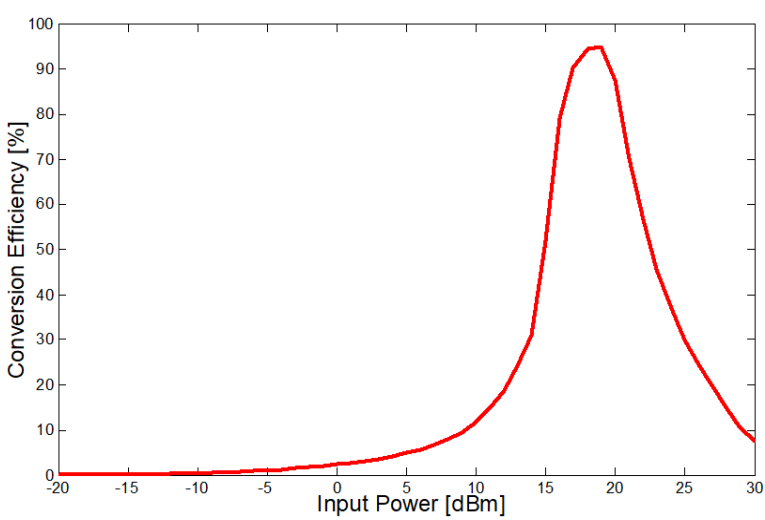

(b)

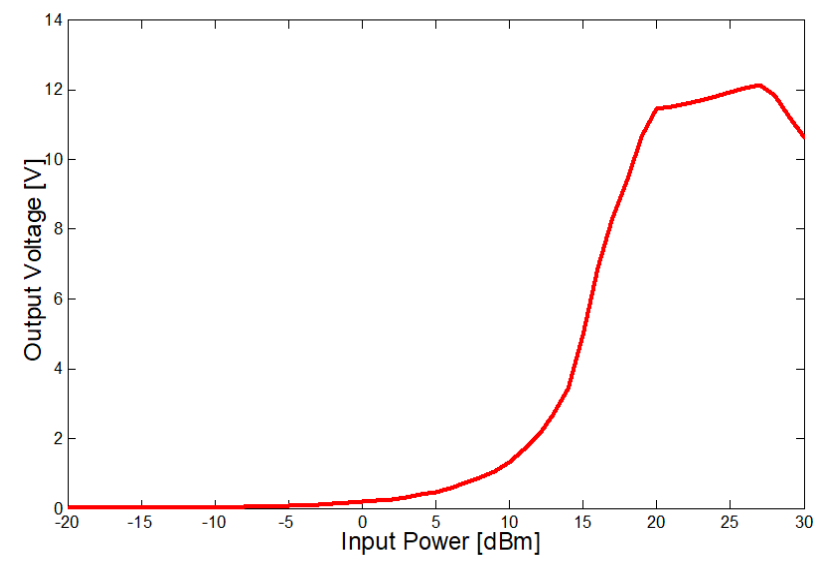

(c)

Figure 9. Simulation results (a) reflection coefficient versus frequency (b) Conversion efficiency versus input power (c) Output voltage versus input power

\section{ACHIEVEMENT AND MEASUREMENTS}

The series-mounted rectifier was built with the intention to measure its performance for different input power points: $-20 \mathrm{dBm}$ to $20 \mathrm{dBm}$. It was printed on an FR4 substrate with dielectric constant of 4.4 and having a thickness of $1.6 \mathrm{~mm}$. The structure of the rectifier is shown in Figure 10; it consists of a Schottky diode in series with a filter at the output of the circuit to remove the unwanted RF component at the load level, this later was chosen to be $2 \mathrm{kOhm}$. For the design and realization of the rectifier, we remind that we have used a Schottky diode type HSMS-2820. The measurement setup shown in Figure 10(a) was used for the experimental characterization of the conversion circuit. It contains an RF generator ANRITSU 68347C $10 \mathrm{MHZ}-20 \mathrm{GHZ}$ to supply the circuits with the RF signal at the desired frequency and a multimeter to measure the output voltage level of the circuit. Figure 11 shows the measured output voltage level versus simulated one, an interesting value is observed with just one rectification diode, this results show the possibility of using our rectifier in real applications on $2.45 \mathrm{GHz}$ of the ISM band. Table 2 shown as performance comparison with other recent researches. 


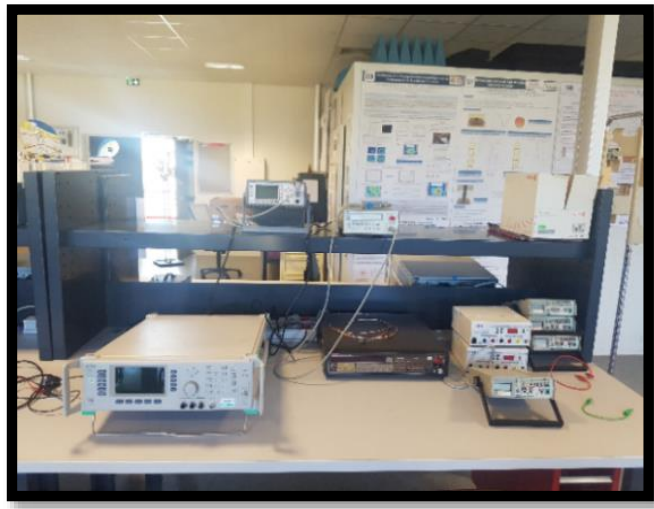

(a)

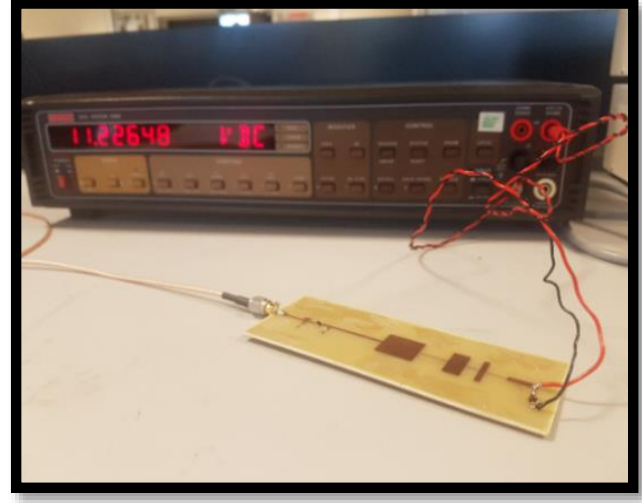

(b)

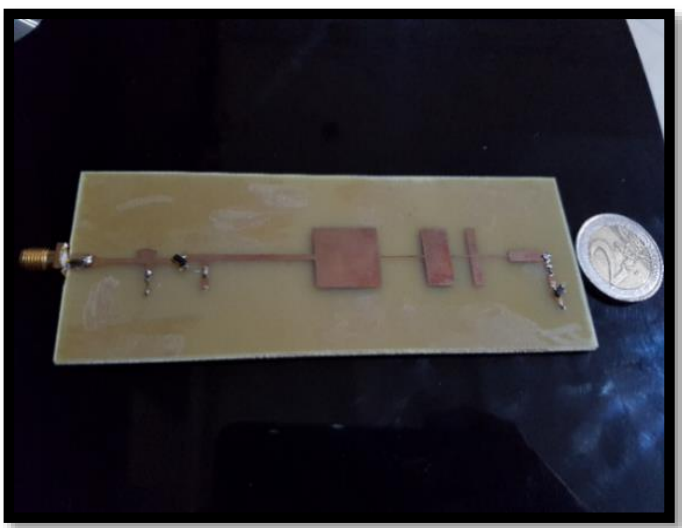

(c)

Figure 10. (a) Measurement setup (b) Measured output voltage (c) Fabricated rectifier

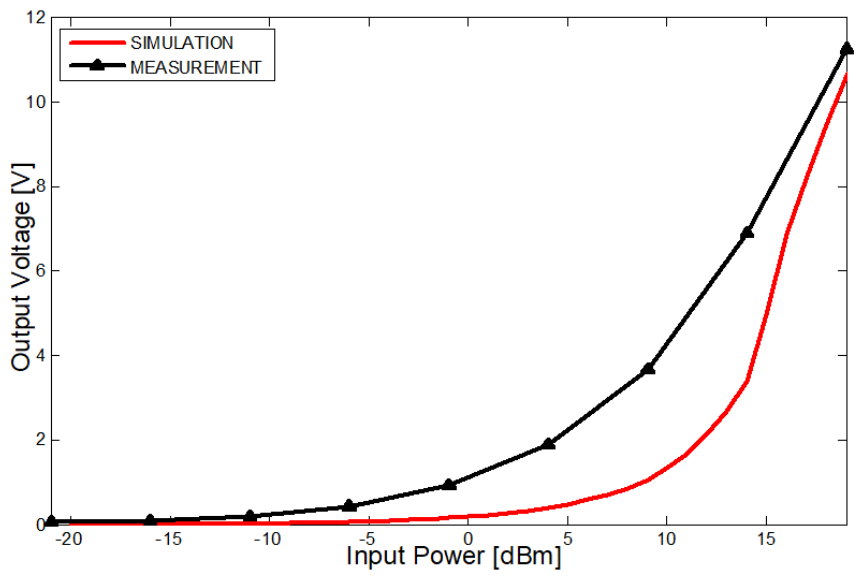

Figure 11. Simulated and measured output voltage versus input power of the rectifier

Table 2. Performance Comparison with Other Recent Researches

\begin{tabular}{cccc}
\hline Source & Operating frequency & Technology & Output Voltage \\
\hline$[3]$ & $2.45 \mathrm{GHz}$ & Voltage Doubler & $10.75 \mathrm{~V}(20 \mathrm{dBm})$ \\
{$[19]$} & $2.45 \mathrm{GHz}$ & Voltage Doubler & $2.3 \mathrm{~V}(\mathrm{Max})$ \\
This paper & $2.45 \mathrm{GHz}$ & Single Diode & $11.23 \mathrm{~V}(20 \mathrm{dBm})$ \\
\hline
\end{tabular}




\section{CONCLUSION}

A high voltage rectifier has been presented to cover the needs for applications involving wireless power transmission. We have used an HSMS2820 Schottky diode as a rectification device mounted in series topology. A stepped-impedance low-pass filter have been used at the output of the circuit to enhance the performance of the rectifier by providing a successful suppression of undesired RF components. Simulations have been carried out by using Advanced Design System and the results have been confirmed by fabrication and measurements. We have used an FR4 substrate with dielectric permittivity constant 4.4, thickness of 1.6 $\mathrm{mm}$ and loss tangent of 0.025 . We have reached an important value of output voltage of $11.23 \mathrm{~V}$ with just one rectification diode, so that the circuit is judged to be compact, low cost and efficient.

\section{REFERENCES}

[1] M. Sudou, H. Takao, K. Sawada, and M. Ishida. A novel RF induced power supply system for monolithically integrated ubiquitous micro sensor nodes. Sensors and Actuators A. 2008; 145-146: 343-348.

[2] C. Yu, F. Tan, and C. Liu. A C-band microwave rectenna using aperture-coupled antenna array and novel Class-F rectifier with cavity. J. Electromag. Waves App. July 2015; 29(8): 977-991.

[3] D. H. Chuc and B. G. Duong. Design and Fabrication of Rectifying Antenna Circuit for Wireless Power Transmission System Operating at ISM Band. International Journal of Electrical and Computer Engineering (IJECE). August 2016; 6(4): 1522-1528.

[4] Deepak Kumar, Kalpana Chaudhary. High Efficiency Harmonic Harvester Rectenna for Energy Storage Application. International Journal of Power Electronics and Drive System (IJPEDS). March 2018; 9(1): 252-259.

[5] Nicolas Tesla. Experiments with Alternate Current of High Potential and High Frequency. London: McGraw, 1904.

[6] W. C. Brown. The history of power transmission by radio waves. IEEE Trans. Microw. Theory Techn. Sep. 1984; MTT-32(9): 1230-1242.

[7] A. Taybi, A. Tajmouati, J. Zbitou, A. Errkik, M. Latrach, L. El Abdellaoui. A New Configuration of a High Output Voltage $2.45 \mathrm{GHz}$ Rectifier for Wireless Power Transmission Applications. Telecommunication, Computing, Electronics and Control Journal (TELKOMNIKA). October 2018; 16(5): 1939-1946.

[8] Kuldeep Kumar Parashar. Design and Analysis of ISlotted Rectangular Microstrip Patch Antenna for Wireless Application. International Journal of Electrical and Computer Engineering (IJECE). 2014; 4(1): 31-36.

[9] C. Y. D. Sim, M. H. Chang, and B. Y. Chen. Microstrip-fed ring slot antenna design with wideband harmonic suppression. IEEE Trans. Antennas Propag. Sep. 2014; 62(9): 4828-4832.

[10] R. A. Rahim, S. I. S. Hassan, F. Malek, M. N. Junita, H. F. Hassan, S. F. W. Anwar. A harmonic suppression circularly polarized patch antenna for an RF ambient energy harvesting system. in Proc. IEEE Int. Conf. Clean Energy Technol. Lankgkawi, Malaysia. Nov. 18-20, 2013: 4933-4936.

[11] Fu-Jhuan Huang, Tzong-Chee Yo, Chien- Ming Lee, and Ching Hsing Luo. Design of Circular Polarization Antenna With Harmonic Suppression for Rectenna Application. IEEE Antennas Wireless Propag. Lett. 2012; 11: $592-595$

[12] Surface mount RF Schottky barrier diode HSMS-282X series. Agilent Technol., Palo Alto, CA, Tech. Data, 2001.

[13] V.Marian, C.Menudier, M.Thevenot, C.Vollaire, J.Verdier, B.Allard. Efficient design of rectifying antennas for low power detection. International Microwave Symposium. Baltimore, United States. Jun 2011: CD.

[14] James O. McSpadden, L. Fan, and K. Chang. Design and experiments of a high-conversion-efficiency 5.8-GHz rectenna. IEEE Antennas. Wireless. Prog. Lett. Dec 1998; 46(12): 2053-2060.

[15] Y.-J Ren and K. Chang. 5.8-GHz Circularly Polarized Dual-Diode Rectenna and Rectenna Array for Microwave Power Transmission. IEEE Trans. On Microwave Theory and Techniques. April 2006; 54(4): 1495-1502.

[16] Jia-Shen G. Hong \& M.J. Lancaster. Microstrip Filters for RF/Microwave Applications. New York: John Wiley \& Sons Inc., 2001.

[17] David M. Pozar. Microwave Engineering. Fourth Edition. John Wiley \& Sons Inc., 2011.

[18] J. Zbitou, M. Latrach and S. Toutain. Hybrid Rectenna and Monolithic Integrated Zero-Bias Microwave Rectifier. IEEE Trans. On Microwave Theory and Techniques. Jan. 2006; 54(1): 147-152.

[19] B.H. Zhang, J.W. Zhang, Z.P. Wu, C.G. Liu and B. Zhang. A 2.45 GHz Dielectric Resonator Rectenna for Wireless Power Transmission. 2017 International conference of Electronics, Communication and Aerospace Technology (ICECA). Coimbatore, India. 20-22 April, 2017. 